\title{
Suddenly moving large classes online: Illuminating the experience of the teaching staff in one university
}

\author{
Mark Anthony Glynn, Ann Marie Farrell, Karen Buckley, Rob Lowney, Sean Smyth, \\ Suzanne Stone \\ Dublin City University, Ireland.
}

\begin{abstract}
In early 2020, the transition of large classes from the face-to-face to the online context occurred overnight and at scale at a time when the crisis was being faced at all levels of society, nationally and internationally.

This paper is based on research which examined the impact of this sudden transition on large classes in Dublin City University with a view to illuminating the experience to inform future practice (Authors., in press). A rapid, systemised review of literature was carried out with the aim of contextualising data gathered through surveys with staff and students in relation to our experience of moving large classes online in the early stages of the Covid-19 pandemic. While the study examined the impact from the perspectives of teaching staff and students, this paper reports on the perspectives of teaching staff only. Large class teachers found this experience challenging, reporting a sense of isolation and worry. However, it would seem that opportunity was seen in the face of adversity, whereby staff have identified potential for better ways of doing things going forward as a result of their experiences between March and May 2020
\end{abstract}

Keywords: Large-classes; online; emergency-teaching; Covid19. 


\section{Introduction}

Technology-enhanced learning (TEL) and a greater flexibility in programme delivery are highlighted as indicators of success/progress in Dublin City University's (DCU) strategic plan 2017-2022 (DCU, 2017). The swift pivot of face-to-face programmes to the online environment as a result of Covid-19 created a situation whereby students and staff had to engage with a teaching and learning context reliant on technology. The transition of classes from the face-to-face to the online context occurred overnight and at scale at a time when the crisis was being faced at all levels of society, nationally and internationally. Therefore, online teaching and learning in this context could not be the same as planned online courses because the former was reactive in nature at a time of crisis while the latter is usually underpinned by evidence based best practices regarding online pedagogy (Eaton, 2020; Hodges et al., 2020) . These practices include development of social, teacher and cognitive presence through carefully designed engagement pathways and activities (Ní Shé et al., 2019); embedding universal design for learning (UDL) to enhance inclusion of all learners online (Kumar \& Wideman, 2014); and the provision of appropriate professional development for teachers embarking on the online teaching journey (Farrell et al., 2020) .

Teaching staff have, by default, learned new skills and been forced to (re)consider pedagogy. At the early stages of the pandemic, teaching staff were forced to fashion solutions quickly and under pressure (Hodges et al., 2020); a unique situation which was swift, often underresourced and characterised by lack of planning (Smith \& Hornsby, 2020). The acquisition of online teaching skills was rapid and at scale as the majority of classes, programmes and modules transitioned online simultaneously. For large class cohorts, the renewed focus on pedagogical practice awakened by the pandemic is welcome, challenging assumptions and providing an opportunity to reimagine the experience of teaching and learning for large groups (Authors., in press). The problems with class size are not embedded in the scale per se but rather in the pedagogical approaches chosen (Hornsby, 2020). Basic pedagogical principles such as good planning, being systematic, developing authentic assessment practices are important as much for teaching large classes (Exeter et al., 2010) as they are for teaching online.

\section{Methodology}

The purpose of this research project was:

- To shed light on the sudden transition of DCU's large classes (100+ students) from the face-to-face teaching and learning setting to the online environment in March 2020 from the perspectives of staff and students. 
- To contextualise the findings arising from the examination of the transition referred to above by reviewing relevant literature.

- To inform the work (a) of academics in DCU teaching large-class cohorts and (b) of DCU's academic developers and learning technologists, supporting them in this endeavour, as large programmes and modules move online in the forthcoming academic year.

The research approach was evaluative in nature, with the aim of illuminating the innovation (Parlett \& Hamilton, 1972) of moving large classes online from a range of perspectives in the DCU context and in the wider, international context. Data were collected from three key sources: (1) a rapid, systematised review of relevant literature; (2) surveys of DCU students and staff who experienced the recent pivot of their large classes online; and (3) information relating to the Teaching Enhancement Unit (TEU) teaching supports provided to staff during the period March-June 2020. The scope of the project was determined by the tight timeline for the project itself and by the urgency of providing guidance by September at the latest. so that it could be used by DCU staff as they moved large-class modules online for the new semester (January 2021).

\subsection{Literature Review}

From the outset, reviewing the literature was conceptualised as a data gathering process, informing the research questions rather than an exercise in providing a rationale for the study per se. To that end, the approach to reviewing the literature was systematic, although due to time the constraints of (a) the project itself and (b) the phenomenon under investigation, it was not possible to conduct a systematic review in its purest sense. The principles underpinning the literature review were as follows:

- To gather together and examine what was already known, understood and experienced by others (Gough et al., 2021) in relation to each element of the focus of this study i.e. large classes, online teaching and learning and inclusion of all learners.

- To gather a range of perspectives to gain an understanding of how the two key pedagogical phenomena under investigation (large classes and the sudden pivot to online learning) were experienced by others and how they approached the challenges arising (Gough et al., 2021). This was particularly important in relation to the sudden pivot online, hence we searched for material through the more unconventional social media route, Twitter to ensure we were accessing a range of perspectives. 
- To utilise a systematic approach to searching the literature which would minimise bias while simultaneously providing clarity (Cornish, 2015), taking the tight timeframe into account.

- To balance the more formulaic systematised searching approach with the interpretive voice of the authors (Booth et al., 2021).

In terms of the swift pivot from face-to-face to online teaching and learning experienced globally by the higher education community since March, it was deemed likely that there would be little value in looking for 'evidence' of best practice in that circumstance but rather, the value in reviewing the literature was to take account of the context and complexity of the situation (Cornish, 2015).

The approach finally adopted can best be described as a combination of a rapid and a systematised review; the completeness of the former determined by time constraints and the latter characterised by a systematic process, stopping short of a systematic review (Grant \& Booth, 2009). This rapid, systemised review was conducted between 15 July and 30 August 2020, focusing on the following key areas:

- Large classes in higher education

- Inclusive approaches to designing learning

- Perspectives on the recent and sudden pivot from the face-to-face to online teaching and learning context

- Existing key DCU research reports relevant to the focus of this study

\subsection{Staff and student survey design}

Staff were surveyed using a questionnaire comprising open and closed questions. The research team decided to use questionnaires to maximise reach to the staff and student body to ensure inclusion of as many perspectives as possible. Questionnaires for both staff and students were developed between May and June. Both versions were piloted with two staff and two students respectively; time did not allow for inclusion of more pilot participants. Feedback from the pilot participants was used to amend and refine aspects of each questionnaire. Google Forms was used as the platform for the questionnaire.

\subsection{Teaching supports}

Both the self-paced resources from our staff support page and the synchronous sessions involve the use of electronic systems that capture data about those that engage with them. As the TEU is responsible for the provision of these resources and offerings, these data were explored to enhance the synthesis of the data arising from the surveys. 


\section{Findings \& Discussion}

Findings are reported from the perspectives of staff only for the purposes of this paper. Full report on findings may be found in the research report (Farrell et al., in press).

Table 1. Class sizes of staff respondents.

\begin{tabular}{cc}
\hline $\begin{array}{l}\text { What best describe the size } \\
\text { of your large class(es)? }\end{array}$ & Staff \\
\hline $100-200$ students & 29 \\
$201-300$ students & 7 \\
$301-400$ students & 2 \\
More than 400 & 9 \\
Total & 47 \\
\hline
\end{tabular}

47 out of 350 staff that teach large classes responded to the survey. Only three of the 47 staff respondents indicated that they had not used Moodle, the university VLE, before moving online in March. Fifty-five per cent $(\mathrm{n}=26)$ staff respondents reported having prior experience of teaching online; however, the depth and breadth of that experience was not explored in the survey.

Using a Likert scale, staff were asked to indicate their comfort levels in terms of teaching online with $73 \%(n=34)$ indicating higher levels of worry about teaching their large classes online at the beginning of the emergency transition. Perhaps unsurprisingly, 70\% $(n=33)$ indicated that they lacked confidence about teaching their large classes online, with at least half of these identifying as being at the lower end of the scale. Interestingly, despite feeling worried and unconfident, staff indicated that they did feel competent to teach their large classes online with 40 staff respondents indicating feeling competent in their ability to carry out their work in the new environment. However, they did indicate that they were more worried about moving their large class cohorts online than they were about smaller groups with $89 \%(\mathrm{n}=42)$ indicating medium to high concerns.

When asked if using Zoom was a challenge, $74 \%(n=35)$ disagreed that it was, with 26 of these respondents strongly disagreeing. This seems to indicate that the use of Zoom as the 'classroom' was not a key factor in contributing to the challenge experienced by staff in terms of synchronous teaching. The perceived lack of challenge in using Zoom may possibly be attributed to the support staff received in using the platform; $78 \%(\mathrm{n}=37)$ disagreed that lack of support was a challenge although $80 \%(\mathrm{n}=38)$ indicated that finding the time to attend training sessions was challenging. $85 \%$ of staff revealed a very strong feeling of disconnect with the student cohort and it may be likely that this is a factor relating to the perception of staff that they struggled with synchronous teaching. At least two-thirds of staff strongly agreed that transferring tasks online was a key challenge. 
Staff views mirrored those of students in terms of their perception that the main advantages of online learning were the provision of additional online resources $(36 \%, n=17)$ and the opportunity for students to engage with materials asynchronously $(21 \%, n=10)$. Some staff also reported that the chat function enabled greater participation with the large class cohort.

Staff were asked open questions regarding what elements of teaching they believed remained the same and what changed when they moved their large classes online. Table 2 provides an overview of the key themes emerging from the analysis of that qualitative data.

Table 2. Staff perceptions of changed and unchanged elements of teaching large classes online.

\begin{tabular}{lclc}
\hline $\begin{array}{l}\text { Unchanged elements of teaching } \\
\text { large classes online }\end{array}$ & & $\begin{array}{l}\text { Changed elements of teaching } \\
\text { large classes online }\end{array}$ & \\
\hline Content being covered & 34 & Less personal interaction & 24 \\
Presentation & 13 & $\begin{array}{l}\text { Harder to adapt delivery to } \\
\text { students' needs }\end{array}$ & 22 \\
Very few similarities & 5 & Student participation & 21 \\
Getting students engaged & 4 & Less student participation & 9 \\
\hline
\end{tabular}

Those staff members teaching large classes did not engage as much with Zoom LSSP resources as staff teaching in other contexts. This is possibly due to the fact that there was more asynchronous engagement planned for large class cohorts than for smaller groups. Furthermore, very large classes (over 300) could not be hosted on Zoom Meetings so all engagement was likely to have been asynchronous for those cohorts. Interestingly, the highest ranked resource was that supporting Loop's Gradebook, indicating perhaps that assessment is a key area requiring staff support regardless of class size. Almost all staff reported that the summative assessment for their large class groups had changed partly (49\%, $\mathrm{n}=23)$ or entirely $(19 \%, \mathrm{n}=9)$ as a result of the move online. With the future in mind staff and students were asked to consider recommendations and suggestions for each of teaching, learning, assessments and technical supports for the next semester. The reason was twofold: acknowledging that Covid-19 would likely still impact in some way at least in the immediate future but also to identify what are the elements of teaching online that can be continued when we return to face to face teaching. Respondents identified a very wide range of areas for improvement. The staff who responded to suggestions regarding learning going forward, tended to make recommendations for their own pedagogical approaches as they had when considering teaching rather than suggesting approaches students might make to enhance their learning in the online space.

\section{Conclusions}

Emergency online teaching is distinctly different to planned online teaching. Despite this research being conducted in relation to the emergency pivot online there is an opportunity 
for DCU to learn from the experiences of the crisis to inform pedagogy (Persky et al., 2020) at institutional, faculty, school, programme and individual academic levels. The feedback from staff and students during this pandemic can help inform both our teaching and our academic staff development going forward.

Staff indicated a sense of disconnect i.e. cameras turned off during synchronous sessions; lack of student engagement, lack of peer interaction generally and specifically in relation to tasks; and lack of feedback on tasks. It would seem that the importance of teachers being explicit in terms of their expectations of synchronous and asynchronous engagement cannot be overemphasized.

Each of these issues can be addressed through application of the community of inquiry model - teacher presence, social presence and cognitive presence (Garrison et al., 1999). The authors would argue that teacher presence in face-to-face teaching of large classes is equally relevant and its importance is often overlooked. However, creating a sense of community and belonging in large classes can be difficult. Students can feel anonymous in large classes, isolated and uncomfortable asking questions in front of their classmates. The major lesson learnt from this research and the recommendation from this research is that the implementation of the same variety of approaches to foster teacher presence in an online environment should be used teaching large classes face to face. The VLE plays a crucial role in implementing the community of inquiry model and as such the second recommendation is that sufficient CPD and support is provided to staff teaching large classes to help optimise the use of the VLE.

In conclusion it would seem that opportunity was seen in the face of adversity, whereby both students and staff have identified potential for better ways of doing things as a result of their experiences between March and May which may be useful going forward, regardless of faceto-face or online contexts. As an institution we must equally see the opportunity and address the concerns raised as part of this research through staff development thereby helping the university meet goals of the university strategic plan.

\section{References}

Booth, A., Sutton, A., \& Papaioannou, D. (2021). Systematic Approaches to a Successful Literature Review (2nd ed.). SAGE publications Ltd. https://uk.sagepub.com/en$\mathrm{gb} /$ eur/systematic-approaches-to-a-successful-literature-review/book244586

Cornish, F. (2015). Evidence synthesis in international development: A critique of systematic reviews and a pragmatist alternative. Anthropology \& Medicine, 22(3), 263-277. https://doi.org/10.1080/13648470.2015.1077199
DCU.
(2017).
Talent,
Discovery
and
Transformation.
DCU. https://www.dcu.ie/sites/default/files/iss/pdfs/web_version_combined.pdf 
Eaton, S. E. (2020). Academic Integrity During COVID-19: Reflections From the University of Calgary. https://prism.ucalgary.ca/handle/1880/112293

Exeter, D. J., Ameratunga, S., Ratima, M., Morton, S., Dickson, M., Hsu, D., \& Jackson, R. (2010). Student engagement in very large classes: The teachers' perspective. Studies in Higher Education, 35(7), 761-775. https://doi.org/10.1080/03075070903545058

Farrell, O., Costello, E., Ní She, C., Brunton, J., Eccles, S., Trevaskis, S., \& Donlon, E. (2020). Openteach Pilot Evaluation Report.

Garrison, D. R., Anderson, T., \& Archer, W. (1999). Critical Inquiry in a Text-Based Environment: Computer Conferencing in Higher Education. The Internet and Higher Education, 2(2), 87-105. https://doi.org/10.1016/S1096-7516(00)00016-6

Gough, D., Oliver, S., \& Thomas, James. (2021). An Introduction to Systematic Reviews (2nd ed.). SAGE publications Ltd. https://uk.sagepub.com/en-gb/eur/an-introduction-tosystematic-reviews/book 245742

Grant, M. J., \& Booth, A. (2009). A typology of reviews: An analysis of 14 review types and associated methodologies. Health Information \& Libraries Journal, 26(2), 91-108. https://doi.org/10.1111/j.1471-1842.2009.00848.x

Hodges, C., Moore, S., Lockee, B., Trust, T., \& Bond, A. (2020). The Difference Between Emergency Remote Teaching and Online Learning. https://er.educause.edu/articles/2020/3/the-difference-between-emergency-remoteteaching-and-online-learning

Kumar, K. L., \& Wideman, M. (2014). Accessible by design: Applying UDL principles in a first year undergraduate course. Canadian Journal of Higher Education, 44(1), 125-147. https://doi.org/10.47678/cjhe.v44i1.183704

Ní She, C., Farrell, O., Brunton, J., Trevaskis, S., Donlon, E., Costello, E., \& Eccles, S. (2019). Teaching online is different: Critical perspectives from the literature.

Parlett, M., \& Hamilton, D. (1972). 'Evaluation as Illumination: A New Approach to the Study of Innovatory Programs'. Occasional Paper. https://eric.ed.gov/?id=ED167634

Persky, A. M., Fuller, K. A., Jarstfer, M., Rao, K., Rodgers, J. E., \& Smith, M. (2020). Maintaining Core Values in Postgraduate Programs During the COVID-19 Pandemic. American Journal of Pharmaceutical Education, 84(6). https://doi.org/10.5688/ajpe8158

Smith, H., \& Hornsby, D. (2020). Towards a Pandemic Pedagogy: Power and politics in learning and teaching. https://doi.org/10.13140/RG.2.2.29280.64005 